\title{
AMELIORATIVE EFFECT OF CAROB AQUEOUS EXTRACT ON WATER PIPE SMOKE INDUCED- TOXICITY IN ADULT MALE ALBINO RATS
}

\author{
MONA ABDEL-RAHMAN, FATMA ELZAHRAA H. SALEM, AMIRA A. BAUOMY, MONA AHMED KHALIFA \\ Zoology Department, Faculty of Science, Helwan University, Cairo, Ain Helwan, 11790 Egypt \\ Email: amiraanwar1@gmail.com
}

Received: 30 Sep 2016 Revised and Accepted: 23 Nov 2016

\section{ABSTRACT}

Objective: The aim of the present study was to investigate the daily effect of pre and post treatment of carob aqueous extract ( $600 \mathrm{mg} / \mathrm{kg}$ b. wt.); on rats exposed to were exposed to water pipe smoke (10 mg/kg; 15 minutes) for 2, 4, 6 and $8 \mathrm{w}$.

Methods: Rats were divided into 6 groups; $1^{\text {st }}$ group was served as a control. Rats of the $2^{\text {nd }}$ group were administered amiodarone (an antiarrhythmic medication used as a model for lung toxicity) at a dose level of $30 \mathrm{mg} / \mathrm{kg}$. The rats of $3^{\text {rd }}$ and $4^{\text {th }}$ groups were exposed to water pipe smoke and treated with carob extract respectively. However, the two remaining groups $\left(5^{\text {th }}\right.$ and $\left.6^{\text {th }}\right)$ were the pre-and post-treatment groups with carob extract respectively.

Results: Amiodarone and water pipe smoke induced a significant increase in serum alanine aminotransferase (ALT), aspartate aminotransferase (AST) and alkaline phosphatase (ALP); on the contrary, induced a significant decrease in serum total protein and albumin. In addition; serum creatinine and urea showed a significant increase; as well as; amiodarone and water pipe smoke increased the oxidative stress (malondialdehyde/glutathione; MDA/GSH) in lung tissue homogenate. While; carob extract gavage showed protective and curative effects in liver and kidney functions; in addition; it decreased MDA level and increased GSH level significantly.

Conclusion: Finally, we can conclude that the carob aqueous extract treatment revealed ameliorative effects on rats were exposed to water pipe smoke.

Keywords: Water pipe smoke, Amiodarone, Carob, Liver, Kidney, Lung, Rat

(C) 2017 The Authors. Published by Innovare Academic Sciences Pvt Ltd. This is an open access article under the CC BY license (http://creativecommons.org/licenses/by/4. 0/] DOI: http://dx.doi.org/10.22159/ijpps.2017v9i1.15470

\section{INTRODUCTION}

The water pipe (shisha, narghile) is a tobacco smoking device. It has long been used in the Middle East, Africa and Asia. Water pipe smoking appears to be more popular among younger people. Moreover, two or more smokers may share the same pipe $[1,2]$

Water pipe smoke produces noxious chemicals: "tar", nicotine, carbon mono-oxide, nitric oxide and various carcinogens [1]. The most common belief among water pipe smoke users across all regions of the world is that water pipe smoke is less harmful and less addictive than cigarette smoking $[3,4]$.

Meanwhile, Cobb et al. [5] suggested that water pipe smoke is associated with cigarette smoking risks. So, water pipe smokers, like cigarette smokers, are at risk for nicotine/tobacco dependence, cardiovascular disease and respiratory tract hazards. Moreover, water pipe smoke induces free radicals accumulation [6].

In addition, smoking induces adverse effects on organs that have no direct contact with the smoke itself such as the liver. Heavy smoking yields toxins, which induce inflammation and increase the severity of hepatic lesions [7]. In the same manner; there is an association between smoking and respiratory tract, lung, stomach, liver, kidneys and urinary tract malignancies [8]. Amiodarone is a potent antiarrhythmic agent, associated with pulmonary fibrosis and hepatotoxicity [9]. Likewise, Gado and Aldahmash [10] reported that amiodarone-induced lung toxicity in rats and it is used as a model for lung toxicity [11].

During the past years; the use of natural products as alternative medicines gained an important among the public, especially medicinal herbs are used in treating some diseases and as protective agents against the toxic effect of some drugs and other toxic compounds [12].

Carob (Ceratonia siliqua L.) is a typical tree of the semiarid environments in the Mediterranean area [13]. It has a long history of the application as a source of health products [14].
The major chemical constituents of the carob are moisture, carbohydrates, proteins, fat, minerals, vitamins, polyphenols and dietary fiber [15]. Polyphenols have anti-oxidative properties which prevent the coronary heart disease and have vasorelaxation effects [16]. In addition carob extract reduces the rate of hepatic synthesis of cholesterol [17]

Since there is no enough information about the effect of carob extract on the toxicity of water pipe smoke. So, the aim of the present study was to investigate the effect of pre-and post-treatment of carob aqueous extract on liver, kidney and lung functions in rats exposed to water pipe smoke by using amiodarone as a model for lung toxicity.

\section{MATERIALS AND METHODS}

\section{Experimental animals}

Adult male albino rats (Rattus rattus) weighing 120-150g, obtained from National Organization for Drug Control and Research. They were housed in polypropylene cages maintained in the laboratory of physiology in Faculty of Science, Helwan University under normal environmental conditions of temperature, humidity and light. They were allowed food on a standard pelled diet (ad libitum). The rats were kept for about one week before experimentation to adapt the laboratory conditions.

\section{Experimental design}

\section{a- Carob (Ceratonia siliqua) pods aqueous extract}

Carob pods were obtained from Egyptian herbal markets, Cairo, Egypt. The pods were grinded and weighted. According to Ayaz et al. [18] the aqueous extract was prepared and evaporated by using rotary evaporator. Rats were received daily oral administration of $600 \mathrm{mg} / \mathrm{kg}$ body weight (b. wt.) [19].

\section{b- Amiodarone}

Amiodarone tablets were obtained from Sanofi-Aventis, Montpellier, France (Commercially found in the form of Cordarone). Rats were received daily oral administration of $30 \mathrm{mg} / \mathrm{kg} \mathrm{b.} \mathrm{wt.} \mathrm{[20].}$ 


\section{c- Water pipe tobacco smoke}

Tobacco (moassal) was obtained from Egyptian stores. Animals were exposed to hookah smoke $(10 \mathrm{gm})$ for 15 minutes [21]. Rats were placed in an isolated box during the experiment in an isolated room and after being exposed to water pipe smoke, the rats were returned to their room. To do this, a special apparatus was designed to have the ability to keep the rats for 15 minutes in a situation very similar to water pipe smoking by a human.

\section{Animal grouping}

\section{Animals were divided into 6 groups (6 animals per group)}

-The $1^{\text {st }}$ group: Rats were received daily oral administration of distilled water for $8 \mathrm{w}$ and served as control.

-The $2^{\text {nd }}$ group (lung toxicity model): Rats were received daily oral administration of amiodarone tablets (30 mg/kg b. wt.) for $8 \mathrm{w}$.

-The $3^{\text {rd }}$ group: Rats were daily exposed to water pipe smoke 10 $\mathrm{mg} / \mathrm{kg}$ b. wt. (15 minutes) for $8 \mathrm{w}$.

-The $4^{\text {th }}$ group: Rats were received daily oral intubation of carob aqueous extract ( $600 \mathrm{mg} / \mathrm{kg} \mathrm{b}$. wt.).

-The $5^{\text {th }}$ group (Protective group): Rats were received daily oral gavage of carob extract $(600 \mathrm{mg} / \mathrm{kg} \mathrm{b}$. wt.) then 30 minutes after rats were exposed to water pipe smoke (15 minutes)

-The $6^{\text {th }}$ group (Therapeutic group): Rats were daily exposed to water pipe smoke (15 minutes) daily then 30 minutes after rats were received daily oral gavage of carob extract $(600 \mathrm{mg} / \mathrm{kg} \mathrm{b}$. wt.).

The experimental duration extended to $8 \mathrm{w}$. The animals were killed by sudden decapitation at different time intervals 2, 4, 6 and $8 \mathrm{w}$ during the experiment. The ethics of animal care and use was followed in this study.

\section{Methods and techniques}

\section{Tissues sampling and biochemical assay}

Rats were sacrificed by sudden decapitation on the $2^{\text {nd }}, 4^{\text {th }}, 6^{\text {th }}$ and $8^{\text {th }}$ weeks. Blood was collected, allowed to stand for 15 minutes, and then centrifuged at $3000 \mathrm{r}$. p. m. for 15 minutes to separate serum and quickly frozen in dry ice $\left(-70{ }^{\circ} \mathrm{C}\right)$ till use for further determination of liver and kidney functions by using Biodiagnostic kits (Biodiagnostic, Dokki, Giza, Egypt).

Serum activities of ALT and AST were determined according to the method of Reitman and Frankel [22]. The activity of ALP was determined by using the method of King and King [23], serum albumin level by the method Kinsley and Frankel [24] and serum total protein level by Lowry et al. [25].
Moreover, serum creatinine and urea levels were determined according to Bartles et al. [26] and Fawcett and Scott [27] respectively. The color intensity was measured in a spectrophotometer.

Lung tissue (40 mg from each rat) was homogenised in ice-cold Tris- $\mathrm{HCl}$ buffer solution PH (7.4) and centrifuged at 2000 r. p. m. for 10 minutes to separate supernatant and quickly frozen in dry ice $\left(-70^{\circ} \mathrm{C}\right)$ till use for malondialdehyde (MDA), reduced glutathione $(\mathrm{GSH})$ determinations.

Briefly; MDA level (the end product of lipid peroxidation) in the lung was determined as thiobarbituric acid reactive substance according to a modification of the method of Ohkawa et al. [28] and the sample absorbance was measured at $535 \mathrm{~nm}$ in a spectrophotometer.

Reduced GSH in homogenate was determined according to Ellman [29]. The procedure is based on the reduction of Ellman's reagent [5, 5 dithiobis-(2-nitrobenzoic acid)] by SH groups to form 1 mole of 2nitro-5-mercaptobenzoic acid per 1 mole of SH. The nitromercaptobenzoic acid anion has intense yellow color and can be determined spectrophotometrically at $412 \mathrm{~nm}$.

\section{Statistical analysis}

The statistical comparisons among the groups were carried out by using one-way ANOVA (Duncan's test); (SPSS version17.0). $\mathrm{P}<0.05$ was considered as significant for all statistical analysis in this study.

\section{RESULTS}

The data tabulated in table (1) showed that amiodarone daily oral administration (30 mg/kg) caused a significant increase in ALT activity in serum all over the experimental periods starting from the $2^{\text {nd }}$ week till the $8^{\text {th }}$ week of the administration recording $32.00 \%, 54.64 \%, 92.11 \%$ and $131.24 \%$ on $2^{\text {nd }}, 4^{\text {th }}, 6^{\text {th }}$ and $8^{\text {th }}$ week respectively as compared to control group. The maximal increase was recorded after $8 \mathrm{w}$.

Similarly, the daily exposure to water pipe smoke $(10 \mathrm{mg} / \mathrm{kg}, \mathrm{b} . \mathrm{wt}$.) for 15 minute induced a significant increase in serum ALT activity after 2, 4, 6 and $8 \mathrm{w}$ with a percentage change $25.72 \%, 41.36 \%$, $78.50 \%$ and $106.00 \%$ respectively as compared to control group. The maximal increase was recorded after $8 \mathrm{w}$. However; the daily oral administration of carob aqueous extract showed a nonsignificant change in the activity of ALT all over the weeks as compared to control group. The pre-treatment with carob extract $(600 \mathrm{mg} / \mathrm{kg})$ for $8 \mathrm{w}$ resulting in a significant increase in serum ALT activity after 2, 4, 6 and $8 w$ recording 16.63\%, 20.00\%, 32.82\% and $26.00 \%$ respectively versus their respective control values. In contrary, ALT activity showed a non-significant change in ALT activity after $2 \mathrm{w}$ and significantly reduction after 4, 6 and $8 \mathrm{w}$ ($15.11 \%,-25.60 \%$ and $-38.78 \%$ respectively) as compared to water pipe smoke group.

Table 1: Effect of daily exposure to water pipe smoke on alanine aminotransferase (ALT) activity in serum of adult male albino rat preand post-treated with carob extract $(600 \mathrm{mg} / \mathrm{kg})$ for $8 \mathrm{w}$

\begin{tabular}{lllll}
\hline Time Groups & $\mathbf{2 ~ W}$ & $\mathbf{4 ~ W}$ & $\mathbf{6 ~ W}$ & $\mathbf{8 ~ W}$ \\
\hline Control & $11.00 \pm 0.68$ & $12.50 \pm 0.43$ & $11.67 \pm 0.67$ & $11.17 \pm 0.48$ \\
Amiodarone & $14.52 \pm 0.52$ & $19.33 \pm 0.71$ & $22.42 \pm 0.80$ & $25.83 \pm 0.60$ \\
& $\left(32.00 \%^{\mathrm{a}}\right)$ & $\left(54.64 \%^{\mathrm{a}}\right)$ & $\left(92.11 \%^{\mathrm{a}}\right)$ & $\left(131.24 \%^{\mathrm{a}}\right)$ \\
Water pipe smoke & $13.83 \pm 0.40$ & $17.67 \pm 0.61$ & $20.83 \pm 0.70$ & $23.00 \pm 0.58$ \\
& $\left(25.72 \%^{\mathrm{a}}\right)$ & $\left(41.36 \%^{\mathrm{a}}\right)$ & $\left(78.50 \%^{\mathrm{a}}\right)$ & $\left(106.00 \%^{\mathrm{a}}\right)$ \\
Carob & $12.00 \pm 0.70$ & $11.33 \pm 0.49$ & $10.33 \pm 0.33$ & $10.07 \pm 0.32$ \\
& $(9.00 \%)$ & $(-9.36 \%)$ & $(-11.50 \%)$ & $(-9.85 \%)$ \\
Carob+water pipe smoke & $12.83 \pm 0.48$ & $15.00 \pm 0.45$ & $15.50 \pm 0.43$ & $14.08 \pm 0.55$ \\
& $\left(16.63 \%^{\mathrm{a}}\right)$ & $(20.00 \% \mathrm{a})$ & $\left(26.00 \% \%^{\mathrm{a}}\right)$ \\
Water pipe smoke+ & $(-7.23 \%)$ & $\left(-15.11 \% \%^{\mathrm{b}}\right)$ & $\left(32.82 \%^{\mathrm{a}}\right)$ & $\left(-38.78 \% 0^{\mathrm{b}}\right)$ \\
Carob & $12.50 \pm 0.56$ & $13.67 \pm 0.56$ & $13.00 \pm 0.93$ & $(12.00 \pm 0.58$ \\
& $(13.63 \%)$ & $(9.36 \%)$ & $(11.40 \%)$ & $(-43 \%)$ \\
\end{tabular}

- mean \pm SE. Statistical analysis performed by using Student's t-test $(n=6)$, a Significant change at $p<0.05$ comparing to control group, bignificant change at $\mathrm{p}<0.05$ comparing to water pipe smoking group, (\%) percentage change.

A non-significant increase in serum ALT activity was observed all over experimental time intervals; as a result of daily carob extract treatment after exposure to water pipe smoke for $8 \mathrm{w}$ (posttreatment) as compared to control group. On the other hand, when 
compared with water pipe smoke group; ALT activity was significantly decreased after 4,6 and $8 \mathrm{w}(-22.64 \%,-37.60 \%$ and$47.83 \%$ respectively).

As shown in table (2); daily amiodarone intubation (at a dose level 30 $\mathrm{mg} / \mathrm{kg} \mathrm{b}$. wt.) indicated a gradual and significant increase $(\mathrm{p}<0.05)$ in serum AST activity all over time intervals with a percentage change $24.25 \%, 30.66 \%, 34.82 \%$ and $67.21 \%$ on the $2^{\text {nd }}, 4^{\text {th }}, 6^{\text {th }}, 8^{\text {th }}$ week respectively versus control group. The maximal increase was recorded after $8 \mathrm{w}$. Likewise, the daily water pipe smoke exposure $(10 \mathrm{mg} / \mathrm{kg}, \mathrm{b}$. wt.) for 15 minutes increased serum AST activity significantly $(18.32 \%, 21.40 \%, 26.40 \%$ and $56.00 \%)$ all over the administered period and the maximal increase was recorded after $8 \mathrm{w}$.

The daily oral gavage of carob aqueous extract induced a nonsignificant increase in serum AST activity all over experimental time intervals. Moreover, a constant and gradual increment occurred in serum AST activity as a result of carob extract pre-treatment recording $13.60 \%, 17.34 \%, 15.20 \%$ and $19.70 \%$ as a percentage change on $2^{\text {td }}, 4^{\text {th }}, 6^{\text {th }}$ and $8^{\text {th }}$ weeks respectively as compared to control values (table, 2).

On the contrary, a significant decrease was occurred in AST activity started from $6^{\text {th }}$ week with a percentage change $(-8.90 \%$ and $-23.30 \%)$ on the $6^{\text {th }}$ and $8^{\text {th }}$ week respectively; versus water pipe smoke group.

In post-treatment group; a constant and significant increase in AST activity started from $4^{\text {th }}$ week till $8^{\text {th }}$ week as compared to control group. Meanwhile, in a comparison to water pipe smoke group a constant and significant reduction was found with a percentage change- $9.50 \%,-9.51 \%,-13.33 \%$ and $-27.00 \%$ on $2,4,6$ and $8 \mathrm{w}$ respectively.

Table 2: Effect of daily exposure to water pipe smoke on aspartate aminotransferase (AST) activity in serum of adult male albino rat preand post-treated with carob extract $(600 \mathrm{mg} / \mathrm{kg})$ for $8 \mathrm{w}$

\begin{tabular}{|c|c|c|c|c|}
\hline Time Groups & $2 \mathrm{~W}$ & $4 W$ & $6 \mathrm{~W}$ & $8 \mathrm{~W}$ \\
\hline Control & $28.17 \pm 0.91$ & $28.83 \pm 0.75$ & $29.67 \pm 0.77$ & $29.80 \pm 0.59$ \\
\hline \multirow[t]{2}{*}{ Amiodarone } & $35.00 \pm 0.73$ & $37.67 \pm 1.20$ & $40.00 \pm 0.58$ & $49.83 \pm 1.25$ \\
\hline & $\left(24.25 \%{ }^{a}\right)$ & $\left(30.66 \%{ }^{a}\right)$ & $(34.82 \% \mathrm{a})$ & $(67.21 \% \mathrm{a})$ \\
\hline \multirow[t]{2}{*}{ Water pipe smoke } & $33.33 \pm 0.71$ & $35.00 \pm 1.15$ & $37.50 \pm 1.07$ & $46.50 \pm 1.26$ \\
\hline & $\left(18.32 \%{ }^{a}\right)$ & $\left(21.40 \%{ }^{a}\right)$ & $\left(26.40 \%{ }^{a}\right)$ & $(56.00 \% \mathrm{a})$ \\
\hline \multirow[t]{2}{*}{ Carob } & $29.67 \pm 1.02$ & $30.17 \pm 0.60$ & $30.50 \pm 0.70$ & $31.20 \pm 0.55$ \\
\hline & $(5.33 \%)$ & $(4.65 \%)$ & $(2.80 \%)$ & $(4.70 \%)$ \\
\hline \multirow[t]{3}{*}{ Carob+water pipe smoke } & $32.00 \pm 0.82$ & $33.83 \pm 1.17$ & $34.17 \pm 1.12$ & $35.67 \pm 1.05$ \\
\hline & $\left(13.60 \%{ }^{a}\right)$ & $\left(17.34 \%{ }^{a}\right)$ & $\left(15.20 \% \%^{a}\right)$ & $(19.70 \% \mathrm{a})$ \\
\hline & $(-4.00 \%)$ & $(-3.34 \%)$ & $\left(-8.90 \%{ }^{b}\right)$ & $\left(-23.30 \% \%^{b}\right)$ \\
\hline \multirow[t]{3}{*}{ Water pipe smoke+Carob } & $30.17 \pm 0.79$ & $31.67 \pm 1.15$ & $32.50 \pm 0.91$ & $34.00 \pm 0.82$ \\
\hline & $(7.10 \%)$ & $\left(9.85 \%{ }^{a}\right)$ & $\left(9.54 \%{ }^{\mathrm{a}}\right)$ & $(14.10 \% \mathrm{a})$ \\
\hline & $\left(-9.50 \%^{\mathrm{b}}\right)$ & $\left(-9.51 \%^{b}\right)$ & $\left(-13.33 \% 0^{\mathrm{b}}\right)$ & $\left(-27.00 \%{ }^{b}\right)$ \\
\hline
\end{tabular}

- mean \pm SE. Statistical analysis performed by using Student's t-test $(n=6)$, a Significant change at $p<0.05$ comparing to control group, bignificant change at $\mathrm{p}<0.05$ comparing to water pipe smoking group, $(\%)$ percentage change.

The level of total protein in serum cleared a gradual, and significant reduction started from $4^{\text {th }}$ week as a result of daily oral gavage of 30 $\mathrm{mg} / \mathrm{kg} \mathrm{b}$. wt. amiodarone treatment and water pipe smoke exposure (10 $\mathrm{mg} / \mathrm{kg}$ b. wt.) for 15 minutes as compared to control value. However, the maximal reduction was observed on $8^{\text {th }}$ week recording$35.34 \%$ and-23.10\% respectively as a percentage change (table, 3 ).

In carob aqueous extract group and the pre-treatment group, there was a non-significant change in total protein level throughout the experimental period versus control value. While, a significant increase in total protein level started from $4^{\text {th }}$ to $8^{\text {th }}$ week in the pretreatment group as compared to water pipe smoke group $(7.86 \%$, $18.20 \%$ and $24.08 \%$ ).

In the same manner, carob extract gavage after water pipe smoke exposure indicated a significant increase in total protein level on $4^{\text {th }}$, $6^{\text {th }}$ and $8^{\text {th }}$ week $(12.43 \%, 27.37 \%$ and $29.03 \%)$ respectively as compared to water pipe smoke group.

Table 3: Effect of daily exposure to water pipe smoke on total protein content in serum of adult male albino rat pre-and post-treated with carob extract $(600 \mathrm{mg} / \mathrm{kg})$ for $8 \mathrm{w}$

\begin{tabular}{|c|c|c|c|c|}
\hline Time Groups & $2 W$ & $4 W$ & $6 \mathrm{~W}$ & $8 \mathrm{~W}$ \\
\hline Control & $7.5 \pm 0.29$ & $8.00 \pm 0.13$ & $7.90 \pm 0.21$ & $8.15 \pm 0.20$ \\
\hline \multirow[t]{2}{*}{ Amiodarone } & $7.00 \pm 0.21$ & $6.43 \pm 0.19$ & $6.00 \pm 0.20$ & $5.27 \pm 0.11$ \\
\hline & $(-6.67 \%)$ & $\left(-19.63 \% \%^{a}\right)$ & $\left(-24.05 \% \%^{a}\right)$ & $\left(-35.34 \% \%^{a}\right)$ \\
\hline \multirow[t]{2}{*}{ Water pipe smoke } & $7.20 \pm 0.29$ & $7.00 \pm 0.28$ & $6.32 \pm 0.20$ & $6.27 \pm 0.18$ \\
\hline & $(-4.00 \%)$ & $(-12.50 \% \mathrm{a})$ & $\left(-20.00 \%{ }^{a}\right)$ & $\left(-23.10 \%{ }^{a}\right)$ \\
\hline \multirow[t]{2}{*}{ Carob } & $7.75 \pm 0.12$ & $8.12 \pm 0.16$ & $8.10 \pm 0.12$ & $8.20 \pm 0.05$ \\
\hline & $(3.33 \%)$ & $(1.5 \%)$ & $(2.53 \%)$ & $(0.61 \%)$ \\
\hline \multirow[t]{3}{*}{ Carob+water pipe smoke } & $7.76 \pm 0.18$ & $7.55 \pm 0.08$ & $7.47 \pm 0.17$ & $7.78 \pm 0.14$ \\
\hline & $(3.46 \%)$ & $(-5.63 \%)$ & $(-5.44 \%)$ & $(-4.54 \%)$ \\
\hline & $(7.78 \%)$ & $(7.86 \%$ b) & $\left(18.20 \%^{\mathrm{b}}\right)$ & $\left(24.08 \%^{\mathrm{b}}\right)$ \\
\hline \multirow{3}{*}{$\begin{array}{l}\text { Water pipe smoke+ } \\
\text { carob }\end{array}$} & $7.25 \pm 0.18$ & $7.87 \pm 0.12$ & $8.05 \pm 0.21$ & $8.09 \pm 0.19$ \\
\hline & $(-3.33 \%)$ & $(-1.63 \%)$ & $(2.00 \%)$ & $(-0.74 \%)$ \\
\hline & $(0.70 \%)$ & $\left(12.43 \%^{\mathrm{b}}\right)$ & $\left(27.37 \%^{\mathrm{b}}\right)$ & $(29.03 \%$ b) \\
\hline
\end{tabular}

- mean \pm SE. Statistical analysis performed by using Student's t-test $(n=6)$, aSignificant change at $p<0.05$ comparing to control group, bignificant change at $\mathrm{p}<0.05$ comparing to water pipe smoking group, \% Percentage change.

There was a significant reduction in serum albumin level started from $6^{\text {th }}$ and $8^{\text {th }}$ week resulting from amiodarone treatment and exposure of rats to water pipe smoke versus its control value (table, 4). Moreover, the daily oral intubation of $600 \mathrm{mg} / \mathrm{kg} \mathrm{b}$. wt. of carob aqueous 
extract induced a significant increase in albumin level on $6^{\text {th }}$ week $(5.76 \%)$ and $8^{\text {th }}$ week $(11.52 \%)$ as compared to control group.

In table (4); a significant decrease in albumin level was occurred on $8^{\text {th }}$ week $(-10.10 \%)$ in the pre-treatment group as compared to control group. Meanwhile, a significant increase was observed on $6^{\text {th }}$ and $8^{\text {th }}$ weeks with a percentage change $9.68 \%$ and $8.33 \%$ respectively as compared to water pipe smoke. In the same manner, a significant increase in albumin level started from $6^{\text {th }}$ week in a post-treatment group versus water pipe smoke group.

Table 4: Effect of daily exposure to water pipe smoke on albumin content in serum of adult male albino rat pre-and post-treated with carob extract $(600 \mathrm{mg} / \mathrm{kg})$ for $8 \mathrm{w}$

\begin{tabular}{lllll}
\hline $\begin{array}{l}\text { Time } \\
\text { Groups }\end{array}$ & $\mathbf{2} \mathbf{W}$ & $\mathbf{4} \mathbf{W}$ & $\mathbf{6 ~ W}$ & $\mathbf{8 ~ W}$ \\
\hline Control & $3.48 \pm 0.05$ & $3.47 \pm 0.06$ & $3.47 \pm 0.02$ & $3.47 \pm 0.03$ \\
Amiodarone & $3.37 \pm 0.08$ & $3.28 \pm 0.06$ & $3.07 \pm 0.03$ & $2.70 \pm 0.09$ \\
& $(-3.16 \%)$ & $(-5.48 \%)$ & $\left(-11.52 \%^{\mathrm{a}}\right)$ & $\left(-22.20 \% \%^{\mathrm{a}}\right)$ \\
Water pipe smoke & $3.40 \pm 0.09$ & $3.30 \pm 0.08$ & $3.10 \pm 0.05$ & $2.88 \pm 0.08$ \\
& $(-2.30 \%)$ & $(-4.90 \%)$ & $\left(-10.66 \%^{\mathrm{a}}\right)$ & $\left(-17.00 \% \%^{\mathrm{a}}\right)$ \\
Carob & $3.43 \pm 0.04$ & $3.52 \pm 0.09$ & $3.67 \pm 0.09$ & $3.87 \pm 0.15$ \\
& $(-1.44 \%)$ & $(1.44 \%)$ & $\left(5.76 \%^{\mathrm{a}}\right)$ & $\left(11.52 \%^{\mathrm{a}}\right)$ \\
Carob+water pipe smoke & $3.40 \pm 0.05$ & $3.42 \pm 0.03$ & $3.40 \pm 0.06$ & $3.12 \pm 0.10$ \\
& $(-2.30 \%)$ & $(-1.44 \%)$ & $(-2.00 \%)$ & $\left(-10.10 \% \%^{\mathrm{a}}\right)$ \\
Water pipe smoke+Carob & $(0 \%)$ & $(3.64 \%)$ & $\left(9.68 \% \%^{\mathrm{b}}\right)$ & $\left(8.33 \%^{\mathrm{b}}\right)$ \\
& $3.42 \pm 0.04$ & $3.47 \pm 0.04$ & $3.45 \pm 0.02$ & $3.40 \pm 0.09$ \\
& $(-1.72 \%)$ & $(0 \%)$ & $(-0.58 \%)$ & $(-2.00 \%)$ \\
\end{tabular}

- mean \pm SE. Statistical analysis performed by using Student's t-test $(n=6)$, aSignificant change at $\mathrm{p}<0.05$ comparing to control group, bignificant change at $\mathrm{p}<0.05$ comparing to water pipe smoking group, \%percentage change.

A gradual and significant increase in serum ALP activity was found as a result of amiodarone treatment started from $2^{\text {nd }}$ till $8^{\text {th }}$ weeks recording $23.00 \%, 45.56 \%, 47.00 \%$ and $62.73 \%$ on $2^{\text {nd }}, 4^{\text {th }}, 6^{\text {th }}$ and $8^{\text {th }}$ week respectively as compared to control group (table, 5). Similarly, the exposure to water pipe smoke induced a significant increase in ALP activity throughout the experimental period; however, ALP activity showed a constant increase on $4^{\text {th }}$ and $6^{\text {th }}$ week $(44.32 \%$ and $44.16 \%)$ respectively and the maximum increase was found on $8^{\text {th }}$ week $(55.00 \%)$.

In pre-treatment group; there was a significant increase in ALP activity on $4^{\text {th }}, 6^{\text {th }}$ and $8^{\text {th }}$ week with a percentage change $(18.00 \%$,
$23.00 \%$ and $30.31 \%$ ) respectively as compared to control group. On the other hand, a significant decrease was noticed on $4^{\text {th }}, 6^{\text {th }}$ and $8^{\text {th }}$ week with a percentage change- $18.27 \%,-14.67 \%$ and- $15.93 \%$ respectively versus water pipe smoke group.

The carob extract gavage after water pipe smoke exposure revealed a significant increase in ALP activity started from $4^{\text {th }}$ week recording $16.00 \%, 17.78 \%$ and $27.34 \%$ on the $4^{\text {th }}, 6^{\text {th }}$ and $8^{\text {th }}$ weeks respectively versus control value.

On the contrary, a significant decrease was cleared in ALP activity on the $4^{\text {th }}, 6^{\text {th }}$ and $8^{\text {th }}$ weeks as compared to water pipe smoke group.

Table 5: Effect of daily exposure to water pipe smoke on alkaline phosphatase (ALP) activity in serum of adult male albino rat pre-and post-treated with carob extract $(600 \mathrm{mg} / \mathrm{kg})$ for $8 \mathrm{w}$

\begin{tabular}{|c|c|c|c|c|}
\hline Time Groups & $2 W$ & $4 W$ & $6 W$ & $8 \mathrm{~W}$ \\
\hline Control & $150.83 \pm 5.69$ & $146.67 \pm 3.80$ & $152.83 \pm 2.87$ & $151.17 \pm 3.52$ \\
\hline Amiodarone & $\begin{array}{l}185.50 \pm 9.48 \\
\left(23.00 \%{ }^{\mathrm{a}}\right)\end{array}$ & $\begin{array}{l}213.50 \pm 5.12 \\
\left(45.56 \%{ }^{a}\right)\end{array}$ & $\begin{array}{l}224.67 \pm 2.06 \\
\left(47.00 \% \%^{\mathrm{a}}\right)\end{array}$ & $\begin{array}{l}246.00 \pm 1.34 \\
\left(62.73 \% \%^{\mathrm{a}}\right)\end{array}$ \\
\hline Water pipe smoke & $\begin{array}{l}180.17 \pm 5.19 \\
\left(19.45 \%^{\mathrm{a}}\right)\end{array}$ & $\begin{array}{l}211.67 \pm 7.24 \\
\left(44.32 \%{ }^{\mathrm{a}}\right)\end{array}$ & $\begin{array}{l}220.33 \pm 3.53 \\
\left(44.16 \%{ }^{\mathrm{a}}\right)\end{array}$ & $\begin{array}{l}234.33 \pm 4.75 \\
\left(55.00 \% \%^{\mathrm{a}}\right)\end{array}$ \\
\hline Carob & $\begin{array}{l}159.50 \pm 2.05 \\
(5.75 \%)\end{array}$ & $\begin{array}{l}153.50 \pm 3.77 \\
(4.66 \%)\end{array}$ & $\begin{array}{l}148.65 \pm 3.59 \\
(-2.74 \%)\end{array}$ & $\begin{array}{l}144.68 \pm 1.89 \\
(-4.30 \%)\end{array}$ \\
\hline Carob+water pipe smoke & $\begin{array}{l}168.67 \pm 6.93 \\
(11.83 \%) \\
(-6.38 \%)\end{array}$ & $\begin{array}{c}173.00 \pm 9.33 \\
\left(18.00 \% \%^{\mathrm{a}}\right) \\
\left(-18.27 \%^{\mathrm{b}}\right)\end{array}$ & $\begin{array}{l}188.00 \pm 3.42 \\
\left(23.00 \% \%^{\mathrm{a}}\right) \\
\left(-14.67 \%^{\mathrm{b}}\right)\end{array}$ & $\begin{array}{l}197.00 \pm 6.73 \\
\left(30.31 \%^{\mathrm{a}}\right) \\
\left(-15.93 \%^{\mathrm{b}}\right)\end{array}$ \\
\hline Water pipe smoke+carob & $\begin{array}{l}164.00 \pm 4.02 \\
(8.73 \%) \\
(-8.97 \%)\end{array}$ & $\begin{array}{l}170.17 \pm 4.87 \\
\left(16.00 \%^{\mathrm{a}}\right) \\
\left(-19.61 \%^{\mathrm{b}}\right)\end{array}$ & $\begin{array}{l}180 \pm 3.17 \\
\left(17.78 \%^{\mathrm{a}}\right) \\
\left(-18.30 \%^{\mathrm{b}}\right)\end{array}$ & $\begin{array}{l}192.50 \pm 5.44 \\
\left(27.34 \%^{\mathrm{a}}\right) \\
\left(-17.85 \%^{\mathrm{b}}\right)\end{array}$ \\
\hline
\end{tabular}

- mean \pm SE. Statistical analysis performed by using Student's t-test $(n=6)$, aSignificant change at $\mathrm{p}<0.05$ comparing to control group, bSignificant change at $\mathrm{p}<0.05$ comparing to water pipe smoking group, \% percentage change.

As shown in table (6); the daily oral administration of amiodarone $(30 \mathrm{mg} / \mathrm{kg})$ caused a significant increase in serum creatinine level all over the experimental periods starting from the $2^{\text {nd }}$ week of the treatment recording $45.71 \%, 108.33 \%, 131.58 \%$ and $148.65 \%$ at $\mathrm{p}<0.05$ as a percentage change on $2^{\text {nd }}, 4^{\text {th }}, 6^{\text {th }}$ and $8^{\text {th }}$ week respectively as compared to control group. The maximal increase was recorded after $8 \mathrm{w}$. likewise; the daily exposure to water pipe smoke $(10 \mathrm{mg} / \mathrm{kg})$ for 15 minutes induced a significant increase in serum creatinine level all experimental time intervals versus control value. However; the maximum increment was noticed after $8 \mathrm{w}$.
The gavage of aqueous extract of carob showed a non-significant change in creatinine level throughout the experimental period. Moreover, in a pretreatment group a significant increase was found in creatinine level started from $4^{\text {th }}$ to $8^{\text {th }}$ week as compared to control group. While; in a comparison to water pipe smoke group a significant decrease was observed in serum creatinine level on $2^{\text {nd }}$, $4^{\text {th }}, 6^{\text {th }}$ and $8^{\text {th }}$ week recording- $18.75 \%,-24.29 \%,-25.64 \%$ and$14.77 \%$ as a percentage change respectively.

The maximum reduction was observed after $6^{\text {th }}$ week. 
The rats were gavaged carob extract after water pipe smoke exposure revealed a gradual and significant increment in creatinine level on $4^{\text {th }}, 6^{\text {th }}$ and $8^{\text {th }}$ week versus the control group.
On the other hand, a gradual and significant decrease was recorded all over time intervals as compared to water pipe smoke group.

Table 6: Effect of daily exposure to water pipe smoke on creatinine content in serum of adult male albino rat pre-and post-treated with carob extract $(600 \mathrm{mg} / \mathrm{kg})$ for $8 \mathrm{w}$

\begin{tabular}{|c|c|c|c|c|}
\hline Time Groups & $2 \mathrm{~W}$ & $4 \mathrm{~W}$ & $6 \mathrm{~W}$ & $8 \mathrm{~W}$ \\
\hline Control & $0.35 \pm 0.02$ & $0.36 \pm 0.02$ & $0.38 \pm 0.01$ & $0.37 \pm 0.02$ \\
\hline \multirow[t]{2}{*}{ Amiodarone } & $0.51 \pm 0.04$ & $0.75 \pm 0.04$ & $0.88 \pm 0.06$ & $0.92 \pm 0.05$ \\
\hline & $\left(45.71 \%{ }^{a}\right)$ & $\left(108.33 \% \%^{\mathrm{a}}\right)$ & $\left(131.58 \%{ }^{\mathrm{a}}\right)$ & $\left(148.65 \%{ }^{a}\right)$ \\
\hline \multirow[t]{2}{*}{ Water pipe smoke } & $0.48 \pm 0.03$ & $0.70 \pm 0.03$ & $0.78 \pm 0.05$ & $0.88 \pm 0.05$ \\
\hline & $\left(37.14 \%{ }^{a}\right)$ & $\left(94.44 \%{ }^{\mathrm{a}}\right)$ & $\left(105.26 \%{ }^{\mathrm{a}}\right)$ & $\left(137.84 \%{ }^{\mathrm{a}}\right)$ \\
\hline \multirow[t]{2}{*}{ Carob } & $0.31 \pm 0.02$ & $0.37 \pm 0.02$ & $0.39 \pm 0.01$ & $0.41 \pm 0.01$ \\
\hline & $(-11.43 \%)$ & $(2.78 \%)$ & $(2.63 \%)$ & $(10.81 \%)$ \\
\hline \multirow[t]{3}{*}{ Carob+wateripe smoke } & $0.39 \pm 0.02$ & $0.53 \pm 0.03$ & $0.58 \pm 0.03$ & $0.75 \pm 0.02$ \\
\hline & $(11.43 \%)$ & $(47.22 \% \mathrm{a})$ & $\left(52.63 \%{ }^{\mathrm{a}}\right)$ & $(102.70 \% \mathrm{a})$ \\
\hline & $\left(-18.75 \%^{b}\right)$ & $\left(-24.29 \% 0^{b}\right)$ & $\left(-25.64 \% \%^{b}\right)$ & $\left(-14.77 \% \%^{b}\right)$ \\
\hline \multirow{3}{*}{$\begin{array}{l}\text { Water pipe smoke+ } \\
\text { carob }\end{array}$} & $0.37 \pm 0.02$ & $0.50 \pm 0.04$ & $0.54 \pm 0.04$ & $0.58 \pm 0.04$ \\
\hline & $(5.71 \%)$ & $(38.90 \% a)$ & $(42.00 \% \mathrm{a})$ & $(56.76 \% \mathrm{a})$ \\
\hline & $\left(-22.91 \%^{\mathrm{b}}\right)$ & $(-28.57 \%$ b $)$ & $(-30.77 \%$ b $)$ & $(-34.09 \%$ b $)$ \\
\hline
\end{tabular}

- mean \pm SE. Statistical analysis performed by using Student's t-test $(n=6)$, a Significant change at $p<0.05$ comparing to control group, bignificant change at $\mathrm{p}<0.05$ comparing to water pipe smoking group, \% percentage change.

Urea level in serum showed a gradual and significant increment resulting from administration of $30 \mathrm{mg} / \mathrm{kg} \mathrm{b}$. wt. of amiodarone on $2^{\text {nd }}, 4^{\text {th }}, 6^{\text {th }}$ and $8^{\text {th }}$ week with a percentage change $20.86 \%, 77.23 \%$, $83.00 \%$ and $104.25 \%$ respectively (table 7 ). The maximum increase occurred on $8^{\text {th }}$ week. Similarly; rats were exposed to water pipe smoke showed a significant increase in serum urea level all overtime period, recording the maximum increase on $8^{\text {th }}$ week with $97.48 \%$ as a percentage change. The rats were intoxicated by water pipe smoke after carob extract gavage showed a significant increase in urea level on $6^{\text {th }}$ week $(18.71 \%)$ and $8^{\text {th }}$ week $(42.00 \%)$ versus control value. On the contrary, a significant decrease was observed on $4^{\text {th }}$ week $(-17.56 \%), 6^{\text {th }}$ week $(-23.56 \%)$ and $8^{\text {th }}$ week $(-28.12 \%)$ versus water pipe smoke group.

Moreover, in post-treatment group urea level showed a significant increase started from $4^{\text {th }}$ till $8^{\text {th }}$ week and the maximum increment was found on $8^{\text {th }}$ week recording $52.12 \%$ as a percentage change as compared to control group.

Meanwhile; a significant decrease was noticed in serum urea level on $4^{\text {th }}, 6^{\text {th }}$ and $8^{\text {th }}$ week with a percentage change- $14.51 \%,-16.44 \%$ and $-22.97 \%$ respectively: versus water pipe group".

Table 7: Effect of daily exposure to water pipe smoke on urea content in serum of adult male albino rat pre-and post-treated with carob extract $(600 \mathrm{mg} / \mathrm{kg})$ for $8 \mathrm{w}$

\begin{tabular}{|c|c|c|c|c|}
\hline $\begin{array}{l}\text { Time } \\
\text { Groups }\end{array}$ & $2 W$ & $4 W$ & $6 \mathrm{~W}$ & $8 \mathrm{~W}$ \\
\hline Control & $25.17 \pm 1.01$ & $35.83 \pm 1.25$ & $39.17 \pm 1.55$ & $39.33 \pm 1.23$ \\
\hline \multirow[t]{2}{*}{ Amiodarone } & $30.42 \pm 1.05$ & $63.50 \pm 2.50$ & $71.67 \pm 1.33$ & $80.33 \pm 2.59$ \\
\hline & $\left(20.86 \%{ }^{\mathrm{a}}\right)$ & $\left(77.23 \%{ }^{\mathrm{a}}\right)$ & $\left(83.00 \%{ }^{a}\right)$ & $\left(104.25 \%{ }^{a}\right)$ \\
\hline \multirow[t]{2}{*}{ Water pipe smoke } & $28.67 \pm 0.99$ & $49.33 \pm 1.31$ & $60.83 \pm 2.25$ & $77.67 \pm 1.50$ \\
\hline & $\left(13.91 \%{ }^{a}\right)$ & $\left(37.68 \%{ }^{a}\right)$ & $\left(55.30 \%{ }^{a}\right)$ & $\left(97.48 \%{ }^{a}\right)$ \\
\hline \multirow[t]{2}{*}{ Carob } & $26.50 \pm 0.52$ & $37.67 \pm 0.84$ & $43.33 \pm 1.22$ & $40.67 \pm 1.36$ \\
\hline & $(5.02 \%)$ & $(5.14 \%)$ & $(10.62 \%)$ & $(3.41 \%)$ \\
\hline \multirow[t]{3}{*}{ Carob+water pipe smoke } & $27.25 \pm 0.93$ & $40.67 \pm 1.36$ & $46.50 \pm 1.76$ & $55.83 \pm 1.08$ \\
\hline & $(8.26 \%)$ & $(13.51 \%)$ & $\left(18.71 \%{ }^{\mathrm{a}}\right)$ & $\left(42.00 \%{ }^{\mathrm{a}}\right)$ \\
\hline & $(-4.95 \%)$ & $\left(-17.56 \%{ }^{b}\right)$ & $\left(-23.56 \%{ }^{b}\right)$ & $\left(-28.12 \%^{b}\right)$ \\
\hline \multirow{3}{*}{$\begin{array}{l}\text { Water pipe smoke+ } \\
\text { carob }\end{array}$} & $27.42 \pm 0.49$ & $42.17 \pm 1.85$ & $50.83 \pm 2.51$ & $59.83 \pm 1.25$ \\
\hline & $(8.94 \%)$ & $(17.70 \% \mathrm{a})$ & $(29.77 \% \mathrm{a})$ & $\left(52.12 \%{ }^{\mathrm{a}}\right)$ \\
\hline & $(-4.36 \%)$ & $\left(-14.51 \%^{b}\right)$ & $\left(-16.44 \% \%^{\mathrm{b}}\right)$ & $\left(-22.97 \%^{\mathrm{b}}\right)$ \\
\hline
\end{tabular}

- mean \pm SE. Statistical analysis performed by using Student's t-test $(n=6)$, aSignificant change at $p<0.05$ comparing to control group, bignificant change at $\mathrm{p}<0.05$ comparing to water pipe smoking group, \% percentage change.

In table (8); pulmonary MDA level showed a significant elevation $(\mathrm{P}<0.05)$ as a result of daily oral administration of amiodarone $(30$ $\mathrm{mg} / \mathrm{kg}$ b.wt.) recording $101.0 \%, 118.0 \%$ and $184.0 \%$ as a percentage change on $2^{\text {nd }}, 4^{\text {th }}$ and $6^{\text {th }}$ week, in addition, a sudden elevation in MDA level was found on $8^{\text {th }}$ week (301.0\%) as compared to control group.

In the same manner, the daily exposure to water pipe smoke (10 $\mathrm{mg} / \mathrm{kg} \mathrm{b}$. wt.) induced a significant increment in pulmonary MDA level on $2^{\text {nd }}, 4^{\text {th }}$ and $6^{\text {th }}$ week $(82.0 \%, 96.73 \%$ and $174.0 \%)$ respectively, moreover; a sudden elevation was occurred in level of pulmonary MDA on the end of the treatment recording $268.0 \%$ as a percentage change versus control value.

In pre-treatment group; the rats exposed to water pipe smoke after carob extract administration showed a significant increment on $4^{\text {th }}$ week $(29.50 \%)$, moreover, on $6^{\text {th }}$ and $8^{\text {th }}$ week there was a sudden and significant elevation in pulmonary MDA level recording $110.0 \%$ and $109.0 \%$ respectively versus control value. Oppositely, in a comparison to water pipe smoke group; there was a significant reduction in MDA level all over the experimental time period. 
However, the carob extract gavage after water pipe smoke exposure (post-treatment) indicated a significant increase $(\mathrm{P}<0.05)$ in MDA level in lung tissue on $2^{\text {nd }}$ and $4^{\text {th }}$ week $(32.87 \%$ and $43.75 \%)$ respectively; in addition; a sudden elevation was observed on $6^{\text {th }}$ and $8^{\text {th }}$ week recording $123.0 \%$ and $140.0 \%$ respectively versus its corresponding control. While, a constant and significant decrease was occurred in MDA level all time intervals versus water pipe smoke group.

Table 8: Effect of daily exposure to water pipe smoke on malondialdehyde (MDA) level in lung tissue of adult male albino rat pre-and post-treated with carob extract $(600 \mathrm{mg} / \mathrm{kg})$ for $8 \mathrm{w}$

\begin{tabular}{|c|c|c|c|c|}
\hline $\begin{array}{l}\text { Time } \\
\text { Groups }\end{array}$ & $2 W$ & $4 W$ & $6 \mathrm{~W}$ & $8 \mathrm{~W}$ \\
\hline Control & $17.22 \pm 0.71$ & $18.65 \pm 0.53$ & $19.86 \pm 0.90$ & $19.33 \pm 0.44$ \\
\hline Amiodarone & $\begin{array}{l}34.64 \pm 0.65 \\
\left(101.00 \%{ }^{a}\right)\end{array}$ & $\begin{array}{l}40.64 \pm 0.98 \\
\left(118.00 \%{ }^{\mathrm{a}}\right)\end{array}$ & $\begin{array}{l}56.49 \pm 1.00 \\
\left(184.00 \%{ }^{\mathrm{a}}\right)\end{array}$ & $\begin{array}{l}77.60 \pm 1.43 \\
\left(301.00 \%{ }^{a}\right)\end{array}$ \\
\hline Water pipe smoke & $\begin{array}{l}31.33 \pm 0.64 \\
\left(82.00 \%{ }^{\mathrm{a}}\right)\end{array}$ & $\begin{array}{l}36.69 \pm 0.83 \\
\left(96.73 \%{ }^{a}\right)\end{array}$ & $\begin{array}{l}54.51 \pm 1.06 \\
\left(174.00 \% \%^{\mathrm{a}}\right)\end{array}$ & $\begin{array}{l}71.13 \pm 2.60 \\
\left(268.00 \% \%^{\mathrm{a}}\right)\end{array}$ \\
\hline Carob & $\begin{array}{l}17.92 \pm 0.82 \\
(4.06 \%)\end{array}$ & $\begin{array}{l}18.10 \pm 0.95 \\
(-2.95 \%)\end{array}$ & $\begin{array}{l}18.38 \pm 0.71 \\
(-7.45 \%)\end{array}$ & $\begin{array}{l}19.10 \pm 0.71 \\
(-1.20 \%)\end{array}$ \\
\hline Carob+water pipe smoke & $\begin{array}{l}18.33 \pm 0.57 \\
(6.45 \%) \\
(-41.50 \% \text { b) }\end{array}$ & $\begin{array}{l}24.15 \pm 1.05 \\
\left(29.50 \%^{\mathrm{a}}\right) \\
\left(-34.18 \%^{\mathrm{b}}\right)\end{array}$ & $\begin{array}{l}41.77 \pm 1.16 \\
\left(110.00 \%^{\mathrm{a}}\right) \\
\left(-23.37 \%^{\mathrm{b}}\right)\end{array}$ & $\begin{array}{l}40.42 \pm 0.89 \\
\left(109.00 \%^{\mathrm{a}}\right) \\
\left(-43.17 \%^{\mathrm{b}}\right)\end{array}$ \\
\hline $\begin{array}{l}\text { Water pipe smoke+ } \\
\text { Carob }\end{array}$ & $\begin{array}{l}22.88 \pm 0.58 \\
\left(32.87 \%^{a}\right) \\
\left(-26.97 \%^{b}\right)\end{array}$ & $\begin{array}{l}26.81 \pm 1.21 \\
\left(43.75 \% \%^{a}\right) \\
\left(-26.93 \% \%^{b}\right)\end{array}$ & $\begin{array}{l}44.29 \pm 0.86 \\
\left(123.00 \%^{\mathrm{a}}\right) \\
\left(-18.75 \%^{\mathrm{b}}\right)\end{array}$ & $\begin{array}{l}46.45 \pm 1.25 \\
\left(140.00 \%^{\mathrm{a}}\right) \\
\left(-34.70 \%^{\mathrm{b}}\right)\end{array}$ \\
\hline
\end{tabular}

- mean \pm SE. Statistical analysis performed by using Student's t-test $(n=6)$, aSignificant change at $p<0.05$ comparing to control group, bSignificant change at $\mathrm{p}<0.05$ comparing to water pipe smoking group, \% percentage change.

The daily oral gavage of amiodarone at a dose level of $30 \mathrm{mg} / \mathrm{kg} \mathrm{b}$. wt. decreased GSH content significantly in lung tissue on 4th, 6th and 8th week with a percentage change- $23.40 \%,-41.00 \%$ and $-48.31 \%$ respectively. Likewise, a significant decrease was recorded resulting from daily exposure to water pipe smoke $(10 \mathrm{mg} / \mathrm{kg} \mathrm{b}$. wt.) for 15 minutes; the maximum reduction occurred on $8^{\text {th }}$ week $(-42.00 \%)$ as shown in the table (9).

Carob aqueous extract gavage $(600 \mathrm{mg} / \mathrm{kg}$ b. wt.) indicated a significant increase in lung GSH content on $4^{\text {th }}, 6^{\text {th }}$ and $8^{\text {th }}$ week with a percentage change $20.53 \%, 33.00 \%$ and $53.68 \%$ respectively.
There was a significant decrease in lung GSH level in pretreatment group started from $4^{\text {th }}$ till $8^{\text {th }}$ week versus control value. While, a non-significant increase was found in GSH content as compared to water pipe smoke group.

The carob extract administration after water pipe smoke exposure (post-treatment group) caused a non-significant change in pulmonary GSH content all over experimental time intervals as compared to control value. Meanwhile, a significant increase was observed in GSH content started from $4^{\text {th }}$ till $8^{\text {th }}$ week versus water pipe smoke group.

Table 9: Effect of daily exposure to water pipe smoke on glutathione (GSH) level in lung tissue of adult male albino rat pre-and posttreated with carob extract $(600 \mathrm{mg} / \mathrm{kg})$ for $8 \mathrm{w}$

\begin{tabular}{lllll}
\hline $\begin{array}{l}\text { Time } \\
\text { Groups }\end{array}$ & $\mathbf{2 ~ W}$ & $\mathbf{4} \mathbf{W}$ & $\mathbf{6 ~ W}$ & $\mathbf{8 ~ W}$ \\
\hline Control & $16.17 \pm 0.33$ & $16.37 \pm 0.87$ & $16.08 \pm 0.33$ & $16.02 \pm 0.52$ \\
Amiodarone & $15.77 \pm 0.34$ & $12.54 \pm 0.51$ & $9.48 \pm 0.39$ & $8.28 \pm 0.30$ \\
& $(-2.47 \%)$ & $\left(-23.40 \%^{\mathrm{a}}\right)$ & $\left(-41.00 \%^{\mathrm{a}}\right)$ & $\left(-48.31 \%^{\mathrm{a}}\right)$ \\
Water pipe smoke & $15.81 \pm 0.29$ & $13.66 \pm 0.67$ & $10.30 \pm 0.36$ & $9.29 \pm 0.41$ \\
& $(-2.22 \%)$ & $\left(-16.60 \%^{\mathrm{a}}\right)$ & $\left(-36.00 \%^{\mathrm{a}}\right)$ & $\left(-42.00 \%^{\mathrm{a}}\right)$ \\
Carob & $17.35 \pm 0.41$ & $19.73 \pm 0.32$ & $21.39 \pm 0.54$ & $24.62 \pm 0.59$ \\
& $(7.30 \%)$ & $\left(20.53 \%^{\mathrm{a}}\right)$ & $\left(33.00 \%^{\mathrm{a}}\right)$ & $\left(53.68 \%^{\mathrm{a}}\right)$ \\
Carob+water pipe smoke & $16.53 \pm 0.81$ & $14.20 \pm 0.34$ & $11.08 \pm 0.35$ & $10.25 \pm 0.43$ \\
& $(2.23 \%)$ & $\left(-13.26 \% \%^{\mathrm{a}}\right)$ & $\left(-31.00 \%^{\mathrm{a}}\right)$ & $\left(-36.00 \% \%^{\mathrm{a}}\right)$ \\
Water pipe smoke+ & $(4.55 \%)$ & $(3.95 \%)$ & $(7.57 \%)$ & $(10.37 \%)$ \\
carob & $16.21 \pm 0.34$ & $15.71 \pm 0.51$ & $15.16 \pm 0.41$ & $15.09 \pm 0.14$ \\
& $(0.25 \%)$ & $(-4.00 \%)$ & $(-5.72 \%)$ & $(-5.80 \%)$ \\
\hline
\end{tabular}

- mean \pm SE. Statistical analysis performed by using Student's t-test $(n=6)$, aSignificant change at $\mathrm{p}<0.05$ comparing to control group, bignificant change at $\mathrm{p}<0.05$ comparing to water pipe smoking group, $\%$ percentage change.

\section{DISCUSSION}

The daily exposure to water pipe smoke (15 minutes) and oral amiodarone $(30 \mathrm{mg} / \mathrm{kg})$ administration increased serum ALT, AST and ALP activities and decreased serum total protein and albumin levels significantly. Moreover, the carob pre-and post-treatment to rats were exposed to water pipe smoke returned the activities of ALT, AST and ALP as well as the levels of serum total protein and albumin to control values. Our results are in agreement with [30-33].
Babatin et al. [34] reported that low cumulative dose of amiodarone-induced acute hepatitis, hepatic failure. Water pipe smoking elevated plasma ALT, AST and ALP activities and it decreased total protein and albumin levels $[32,33]$.

The increased activity of ALP was produced by liver, kidney and bone which is a mainly marker of the liver turnover [35]. In the same manner, change in serum transaminase activity is an indicator of early hepatic inflammation and liver cell damage [36]. 
However, the reduction in plasma protein of smokers could be attributed to the damaging effect of harmful compounds from cigarette smoking on liver cells as confirmed by the increase in the activities of plasma ALT, AST and ALP [37].

Gulay et al. [31] demonstrated that traditional use of carob extract is non-toxic and have no significant adverse effects in rabbits, so serum ALT, AST and ALP activities were within the normal ranges [30].

In the present study the daily oral administration of amiodarone (30 $\mathrm{mg} / \mathrm{kg}$ ) and exposure to water pipe smoke (15 minute) induced an increase in serum creatinine and urea levels, while oral administration of carob before/after water pipe exposure decreased the elevated levels of creatinine and urea in serum significantly.

It was reported that the increased level of serum creatinine was associated with amiodarone therapy [38, 39]; which are in agreement with the present study. In addition, Kannan et al. [40] concluded that amiodarone-induced renal damage in rat.

Hallan and Orth [41] demonstrated that smoking-induced renal failure which increased urea reabsorption [41-43]. Smoking of 20 cigarettes per day results in inhalation of $\mathrm{Cd}$, which is a cumulative nephrotoxicant [44]; which increased tubular proteinuria and induced glomerular dysfunction evidenced by an increased level of creatinine in plasma [45].

The daily oral administration of amiodarone and intoxication by water pipe smoking increased MDA level significantly in pulmonary tissue, on the contrary; level of GSH was reduced significantly. The pre-and post-treatment groups showed that carob indicated a significant decrease in MDA level and a significant increase in GSH level in lung tissue. Our results go hand in hand with that of $[46,47,48,6]$.

Amiodarone has been shown to generate free radicals that may be involved in the pathogenesis of its toxicity [48]

Wolfram et al. [49] reported that a significant increase in MDA, as a biomarker for oxidative stress and a significant decrease in vitamin $\mathrm{C}$ as a potent antioxidant in water pipe smokers were recorded. So, Ben Saad et al. [46] and Sharma et al. [47] emphasised that the regular water pipe smoke increased oxidative stress significantly which leads to an imbalance in the production/consumption level of reactive oxygen species. In the same manner, Al-Awaida et al. [6] deduced that the repeated daily smoking-induced a persistent, long-lasting oxidation injury. Some studies have shown the antioxidant activity of carob which is attributed to the presence of phenolic compounds [50, 51]; mainly due to their redox properties, which can play an important role in absorbing and neutralizing free radicals, quenching singlet and triplet oxygen, or decomposing peroxides [52], so polyphenols enhances the prevention or delay the oxidative damage [53].

Nicotine, a major constituent of tobacco smoke, is mainly metabolised in the liver and induces lesions characterised by focal or confluent necrosis, and varying degrees of fibrosis [54].

\section{CONCLUSION}

From our results and previous studies, it could be concluded that the pre and post-treatment of carob aqueous extract ameliorated the impairments in liver, kidney and lung functions and decreased the oxidative stress that induced by exposure to water pipe smoke and amiodarone in rats. Collectively, carob extract is very promising for the effective phytomedicine; so further studies are required to investigate the bioactive fractions in the carob pods aqueous extract.

\section{CONFLICTS OF INTERESTS}

The authors report no conflicts of interest in this work.

\section{REFERENCES}

1. Akl EA, Gunukula SK, Aleem S, Obeid R, Jaoude PA, Honeine R, et al. The prevalence of water pipe tobacco smoking among the general population and specific populations: a systematic review. BMC Public Health 2011;11:244.

2. Grant A, Morrison R, Dockrell M. The prevalence of shisha (narghille, hookah, water pipe) use among adults in Great
Britain, and factors associated with shisha use. Nicotine Tob Res 2014;16:931-8.

3. Jawad M, Jawad J, Mehdi A, Sardar A, Jawad AM, Hamilton FL. A qualitative analysis among regular water pipe tobacco smokers in London universities. Int J Tuberculosis Lung Disease 2013;17:1364-9.

4. Jawad M, McEwan A, McNeill A, Shahab L. Tow hat extent should water pipe tobacco smoking become a public health priority? Addiction 2013;108:1873-84.

5. Cobb CO, Shihadeh A, Weaver MD, Eissenberg T. Water pipe tobacco smoking and cigarette smoking: a direct comparison of toxicant exposure and subjective effects. Nicotine Tob Res 2011;13:78-87.

6. Sharma P, Kumar P, Sharma R, Kishor K, Gupta G. Dyslipidemia among smokers. Asian J Pharm Clin Res 2016;9:137-8.

7. Al-Easawi NAF, Al-Azzawi MNA. A histological study in the liver of albino mice posts is exposing to shisha smoke. World J Exp Biosci 2015;3:30-5.

8. Liu M, Poo WK, Lin YL. Pyrazine, 2-ethylpyridine, and 3ethylpyridine are cigarette smoke components that alter the growth of normal and malignant human lung cells and play a role in multidrug resistance development. Exp Mol Pathol 2015;98:18-26.

9. Daniels JM, Leeder RG, Brien JF, Massey TE. Repeated amiodarone exposure in the rat: toxicity and effects on hepatic and extrahepatic monooxygenase activities. Can J Physiol Pharmacol 1990;68:1261-8.

10. Gado AM, Aldahmash BA. Protective effect of L-carnitine against amiodarone-induced lung toxicity in rats. Int J Toxicol 2013;10:1-9.

11. Zidan RA. Effect of long-term administration of amiodarone on rat lung and the possible protective role of vitamin E: a histological and immunohistochemical study. Egyptian J Histol 2011;34:117-28.

12. Zhou LA, Zhu XP, Chen ZO, Yin M, Cheng X. Protective effect of bilobalide on amyloid beta-peptide 25-35-induced PC12 cell cytotoxicity. Acta Pharmacol Sin 2000;21:75-9.

13. Biner B, Gubbuk H, Karham M, Aksu M, Pekmeczi M. Sugar profiles of the pods of cultivated and wild types of carob bean (Ceratonia siliqua L.) in Turkey. Food Chem 2007;100:1453-5.

14. Makris DP, Kefalas P. Carob pods (Ceratonia siliqua L.) as a source of polyphenolic antioxidants. Food Technol Biotechnol 2004;42:105-8.

15. Yousif AK, Alghazawi HM. Processing and characterization of carob powder. Food Chem 2000;69:282-7.

16. Sakakibara H, Sakakibal H, Honda Y, Nakagawa S, Ashida H, Kanazawa K. Simultaneous determination of all polyphenols in vegetables, fruits and teas. J Agricul Food Chemist 2003;51:571-81.

17. Ruiz-Roso B, Quintela J, de la Fuente E, Haya J, Pérez-Olleros L. Insoluble carob fiber rich in polyphenols lowers total and LDL cholesterol in hypercholesterolemic subjects. Plant Foods Hum Nutr 2010;65:50-6.

18. Ayaz FA, Torun H, Ayaz S, Correia PJ, Alaiz M, Sanz C, et al. Determination of chemical composition of anatolian carob pod (Ceratonia siliquaL.): sugars, amino and organic acids, minerals and phenolic compounds. Food Qual 2007;30:1040-55.

19. Mokhtary M, Sharifi E, Shahamir-tabatabaee M. The effect of carob extract on liver function test in the diabetic male rat. J Shahrekord Univ Med Sci 2013;15:40-7.

20. Agelaki MG, Pantos C, Korantzopoulos P, Tsalikakis DG, Baltogiannis GG, Fotopoulos $\mathrm{A}$, et al. Comparative antiarrhythmic efficacy of amiodarone and dronedarone during acute myocardial infarction in rats. Eur J Pharmacol 2007;564:150-7.

21. Miri-Moghaddam E, Mirzaei R, Arab MR, Kaikha S. The effects of water pipe smoking on haematological parameters in Rats. Int J Hematol Oncol Stem Cell Res 2014;1:37-43.

22. Reitman S, Frankel A. Colorimetric method for determination of serum glutamate oxaloacetate and glutamic pyruvate transaminase. Am J Clin Pathol 1957;28:56-8. 
23. King PR, King EJ. Estimation of plasma phosphates by determination of hydrolyzed phenol with antipyrine. J Clin Pathol 1954;7:322-6.

24. Kinsley SR. Frankel SJ. The determination of serum total protein and albumin-globulin ratio. J Biol Chem 1939;128:131-7.

25. Lowry $\mathrm{OH}$, Rosenbrough NJ, Farr AL, Randall RJ. Protein measurement with Folin-Phenol reagent. J Biol Chem 1951;193:265-75.

26. Bartles H, Bohmer M, Heirli C. Colorimetric kinetic method for creatinine determination in serum and urine. Clin Chim Acta 1972;27:193.

27. Fawcett JK, Scott JE. A rapid and precise method for the determination of urea. J Clin Pathol 1960;13,156-9.

28. Ohkawa H, Ohishi N, Yagi K. Assay for lipid peroxides in animal tissues by thiobarbituric acid reaction. Anal Biochem 1979;95:351-8.

29. Ellman GL. Tissue sulfhydryl groups. Arch Biochem Biophys 1959;82:70-7.

30. Hein J, Hartmann K. Reference ranges for laboratoy parameters in rabbits. Tieraztliche Praxis Ausgabe Kleintiere Heimtiere 2003;31:321-8.

31. Gulay MS, Yildiz-Gulay 0 , Ata A, Balic A, Demirtas A. Toxicological evaluation of carob (Ceratonia siliqua) bean extracts in male New Zealand white rabbits. J Anim Vet Adv 2012;11:1853-7.

32. Dass BP, Jaganmohan $\mathrm{P}$, Sravanakumar $\mathrm{P}$. Changes in hematological and biochemical parameters in smokeless tobacco (ST) chewers in costal belt of Andhra Pradesh. India Eur J Biol Sci 2013;5:29-33.

33. Foroutanjazi M, Naghavi E, Molaee S, Ahmadi RWI. The Effects of Inactivity with Smoking: High Risk for Heart or Liver Dysfunction in Females International Conference on Chemical, Agri Med Sci; 2014. p. 29-31.

34. Babatin M, Lee SS, Pollak PT. Amiodarone hepatotoxicity. Curr Vasc Pharmacol 2008;6:228-36.

35. Nathwani RA, Pais S, Reynolds TB, Kaplowitz N. Serum alanine aminotransferase in skeletal muscle diseases. Hepatology 2005;41:380-2.

36. Raja MM, Raja A, Imran MM, Santha AM, Devasena K. Enzymes application in diagnostic prospects. Enzymes application in diagnostic prospects. Biotechnology 2011;10:51-9.

37. Alsalhen KS, Abdalsalam RD. Effect of cigarette smoking on liver functions: a comparative study conducted among smokers and non-smokers male in El-beida City, Libya. Int Curr Pharm J 2014;3:291-5.

38. Jacobs MB. Serum creatinine increases associated with amiodarone therapy. N Y State J Med 1987;87:358-9.

39. Pollak PT, Sharma AD, Carruthers G. Creatinine elevation in patients receiving amiodarone correlates with serum amiodarone concentration. Br J Clin Pharmacol 1993;36:125-7.

40. Kannan R, Sarma JS, Guba M, Venkataraman K. Amiodarone toxicity. II. Desethylamiodarone-induced phospholipidosis and ultrastructural changes during repeated administration in rats. Fund Appl Toxicol 1991;16:103-9.
41. Hallan SI, Orth SR. Smoking is a risk factor in the progression to kidney failure. Kidney Int 2011;80:516-23.

42. Ritz E, Benck U, Franek E, Keller C, Seyfarth M, Clorius J. Effects of smoking on renal hemodynamics in healthy volunteers and in patients with glomerular disease. J Am Soc Nephrol 1998;9:1798-804.

43. JoAnn L, Robert WS. Blood urea nitrogen a marker for adverse effects of loop diuretics. J Am Coll Cardiol 2011;58:383-5.

44. Ahmed ME, Jawad AS, Osman HM, Shayoub ME. The effect of smoking cigarette on kidney functions among sundaes peoples. Int J Drug Dev Res 2015;5:4473-5.

45. Roszczenko A, Galazyn-Sidorczuk M, Brzoska MM, MoniuszkoJakoniuk J, Zwierz K. Chosen parameters of the kidney function in smokers in relation to the exposure to cadmium. Przegl Lek 2004;61:348-50.

46. Ben Saad H, Babba M, Boukamcha R, Latiri I, Knani J, Slama R, et al. Submaximal exercise capacity and quality of life in exclusive water-pipe smokers. Rev Mal Respir 2010;27:489-95.

47. Sharma R, Deva C, Behera D. Reactive oxygen species formation in peripheral blood neutrophils in different types of smokers. Indian J Med Res 1997;106:475-80.

48. Ray SD, Patel D, Wong V, Bagchi D. In vivo protection of DNA damage associated apoptotic and necrotic cell deaths during acetaminophen-induced nephrotoxicity, amiodarone-induced lung toxicity and doxorubicin-induced cardiotoxicity by a novel IH636 grape seed proanthocyanidin extract. Res Commun Mol Pathol Pharmacol 2000;107:137-66.

49. Wolfram RM, Chehne F, Oguogho A, Sinzinger H. Narghile (water pipe) smoking influences platelet function and (iso-) eicosanoids. Life Sci 2003;74:47-53.

50. Owen RW, Haubner R, Hull WE, Erben GB, Spiegelhalder H, Bartsch B. Isolation and structure elucidation of the major individual polyphenols in carob fibre. Food Chem Toxicol 2003;41:1727-38.

51. Papagiannopoulos M, Wollseifen HR, Mellenthin A, Haber B, Galensa R. Identification and quantification of polyphenols in carob fruits (Ceratonia siliqua L.) and derived products by HPLC-UVESI/MS. J Agric Food Chem 2004;52,3784-91.

52. El Hajaji H, Lachkar N, Alaoui K, Cherrah Y, Farah A, Ennabili A et al. Antioxidant properties and total phenolic content of three varieties of carob tree leaves from morocco. Rec Nat Prod 2010;4:193-204.

53. Lee CG, Koo JH, Kim SG. "Phytochemical regulation of Fyn and AMPK signalling circuitry”. Arch Pharmacal Res 2015;38:2093-105.

54. Omotoso GO, Enaibe BU, Akinola OB, Kadir RE, Akinlolu AA, Oyewopo AO, et al. Lipid profile and liver histochemistry in animal models exposed to cigarette smoke. J Basic Appl Sci 2012;8:12-7.

\section{How to cite this article}

- Mona Abdel-Rahman, Fatma Elzahraa H Salem, Amira A Bauomy, Mona Ahmed Khalifa. Ameliorative effect of carob aqueous extract on water pipe smoke induced toxicity in adult male albino rats. Int J Pharm Pharm Sci 2017;9(1):246-253. 